\title{
Vortex line density in counterflowing He II with laminar and turbulent normal fluid velocity profiles
}

\author{
A. W. Baggaley, ${ }^{1, \circledast}$ and S. Laizet ${ }^{2, \text { 团 }}$ \\ ${ }^{1}$ School of Mathematics and Statistics, University of Glasgow, Glasgow, G12 8QW, UK \\ ${ }^{2}$ Turbulence, Mixing and Flow Control Group, Department of Aeronautics, \\ Imperial College London, London SWr $2 P G, U K$
}

Superfluid helium is an intimate mixture of a viscous normal fluid, with continuous vorticity, and an inviscid superfluid, where vorticity is constrained to thin, stable topological defects. One mechanism to generate turbulence in this system is through the application of a heat flux, so called thermal counterflow. Of particular interest is how turbulence in the superfluid responds to both a laminar and turbulent normal fluid in the presence of walls. We model superfluid vortex lines as reconnecting space curves with fixed circulation, and consider both laminar (Poiseuille) and turbulent normal fluid flows in a channel configuration. Using high resolution numerical simulations we show that turbulence in the normal fluid sustains a notably higher vortex line density than a laminar flow with the same mean flow rate. We exam Vinen's relation, $\sqrt{L}=\gamma v_{n s}$, between the steady state vortex line density $L$ and the counterflow velocity $v_{n s}$. Our results support the hypothesis that transition to turbulence in the normal fluid is responsible for the TI to TII transition. We also consider the spectral properties of fluctuations of the superfluid vortices, which show a good agreement with previous experimental results.

* andrew.baggaley@gla.ac.uk

$\dagger$ s.laizet@imperial.ac.uk 


\section{INTRODUCTION}

Turbulence, the chaotic, disorder motion of fluids, poses tremendous mathematical, physical and engineering challenges. In recent years a large body of work 1, 2] has highlighted similarities between the turbulence of superfluid helium II (quantum turbulence) and the turbulence of ordinary (viscous) fluids. For example, both experimental [3, 4] and numerical [5 7] studies have shown that the distribution of the superfluid kinetic energy is consistent with the $k^{-5 / 3}$ Kolmogorov scaling observed in classical turbulence. Other experimental studies have also observed Kolmogorov's four-fiths law [8], and quasi-classical behaviour in the decay of quantum turbulence [9, 10]

The similarity is particularly striking as superfluid helium is unlike an ordinary fluid. It possesses a two-fluid nature, indeed the system is an intimate mix of a viscous normal fluid component and an inviscid superfluid component, which are coupled through mutual friction [11]. Perhaps of more interest is that due to the constraints of quantum mechanics, the superfluid vorticity field is not continuous. Instead it is restricted to discrete vortex filaments around which the circulation is fixed to the ratio of Planck's constant and the mass of one helium atom. The commonly held definition of quantum turbulence is the motion induced by a tangle of these quantised vortices. Hence, quantum turbulence may provide a simplified model, a 'skeleton', where one can hope to learn more about the complex mechanisms present in classical turbulence.

Whilst the similarities between quantum and classical turbulence are striking, there are other forms of quantum turbulence which are unique to this two-fluid system. One of these, thermal counterflow [12], is the subject of this study. This form of turbulence is typically created by heating one end of a channel containing superfluid helium. The heat flux is carried from the heater via the normal fluid alone and, due to conservation of mass, the normal fluid and the superfluid move in opposite directions; their velocity difference is proportional to the applied heat flux. This was the setting for the earliest studies of quantum turbulence [13 [16], and still poses a number of open questions, which continue to attract experimental [9, 17] and theoretical studies [18, 19].

It is important to stress that although counterflow has no classical analogy, it does not necessarily mean that the study of counterflow cannot offer deeper insights into phenomena in classical turbulence. For example Skrbek et al. 9] emphasised that the efficiency of turbulent heat transport in counterflow is similar to that of turbulent thermal convection in a classical fluid. Of particular interest in recent years has been the question of the transition from laminar to turbulent flow in the normal fluid component [20]. One would expect that a deeper understanding of this transition would be important when considering the transition to turbulence in a classical fluid.

This transition to turbulence is also believed to be important in understanding the different turbulent states observed in thermal counterflow. In channels with a small (cross-sectional) aspect ratio, Tough [12] categorised two turbulent regimes TI and TII, which exhibit strong difference in the scaling of the steady state vortex line density to the applied heat flux. Stability analysis by Melotte\& Barenghi [21] indicated that transition to turbulence in the normal fluid was the underlying reason for the transition from the TI to TII regime. Fully probing this transition is beyond the scope of this current study; our goal here is to better understand differences in the structure of the quantised vortices when the normal fluid is laminar (Poiseuille) and turbulent. To this end we perform high resolution numerical simulations using the vortex filament method and compare and contrast statistical information about the steady state tangles at two different temperatures.

In the next section we discuss our approach to numerically modelling the evolution of quantised vortices (section II). Section III is devoted to details about the numerical modelling of a turbulent normal fluid. Section IV] summarises our findings, focusing in particular on the structure of the vortex tangle, as well as fluctuations of the vortex line density. Finally we close with brief comments and conclusions in section V]

\section{THE VORTEX FILAMENT METHOD}

Following Schwarz [22], we model quantum vortex filaments as space curves $\mathbf{s}(\xi, t)$ which move according to

$$
\frac{d \mathbf{s}}{d t}=\mathbf{v}_{s}+\alpha \mathbf{s}^{\prime} \otimes\left(\mathbf{v}_{n}-\mathbf{v}_{s}\right)-\alpha^{\prime} \mathbf{s}^{\prime} \otimes\left(\mathbf{s}^{\prime} \otimes\left(\mathbf{v}_{n}-\mathbf{v}_{s}\right)\right)
$$

where $t$ is time, $\alpha$ and $\alpha^{\prime}$ are temperature dependent friction coefficients [23], $\mathbf{s}^{\prime}=d \mathbf{s} / d \xi$ is the unit tangent vector at the point $\mathbf{s}, \xi$ is arc length, and $\mathbf{v}_{n}$ is the externally applied normal fluid velocity. We perform all calculations in the frame of reference of the imposed superflow, hence the total superfluid velocity $\mathbf{v}_{s}$ reduces to the self-induced velocity of the vortex filament, which is given by the Biot-Savart law

$$
\mathbf{v}_{s}(\mathbf{s})=-\frac{\Gamma}{4 \pi} \oint_{\mathcal{L}} \frac{(\mathbf{s}-\mathbf{r})}{|\mathbf{s}-\mathbf{r}|^{3}} \times \mathbf{d r}
$$


where $\Gamma=9.97 \times 10^{-4} \mathrm{~cm}^{2} / \mathrm{s}$ is the quantum of circulation and the line integral extends over the entire vortex configuration $\mathcal{L}$.

In numerical studies of thermal counterflow [18, 24, 25], it is common to simplifying the problem by taking a uniform velocity profile for $\mathbf{v}_{n}$, neglecting the important effect of boundaries. In addition no numerical study has investigated the role turbulence in the normal fluid may have on the system. In this study we take a simple analytic Poiseuille profile, suitable for a laminar normal fluid in a channel. This is contrasted with a turbulent velocity field, obtained by numerically solving the incompressible Navier-Stokes equations. Both velocity fields will be described in more detail below, however it is important to state here that in all cases the normal fluid is simply prescribed, ignoring any modification of the flow due to the presence of the quantised vortices. While this is clearly sub-optimal, a dynamically self-consistent model would be very complex and computationally expensive. Indeed an imposed normal fluid is still the most common approach in the literature [18, 26 28], and a better understanding of key physical processes can be garnered from such an approach.

The evolution of a system of vortex filaments, is achieved numerically by discretizing continuous space curves, $\mathbf{s}(\xi, t)$, into a large number of points $\mathbf{s}_{i}(i=1, \cdots N)$. The singularity at $\mathbf{s}=\mathbf{r}$ is removed in a standard way by considering local and non-local contributions to the integral. If $\mathbf{s}_{i}$ is the position of the $i^{\text {th }}$ discretization point along the vortex line, Eq. (2) becomes [22]

$$
\frac{d \mathbf{s}_{i}}{d t}=\frac{\Gamma}{4 \pi} \ln \left(\frac{\sqrt{\ell_{i} \ell_{i+1}}}{a}\right) \mathbf{s}_{i}^{\prime} \otimes \mathbf{s}_{i}^{\prime \prime}-\frac{\Gamma}{4 \pi} \oint_{\mathcal{L}^{\star}} \frac{\left(\mathbf{s}_{i}-\mathbf{r}\right)}{\left|\mathbf{s}_{i}-\mathbf{r}\right|^{3}} \times \mathbf{d r} .
$$

Here $\ell_{i}$ and $\ell_{i+1}$ are the arclengths of the curve between points $\mathbf{s}_{i-1}$ and $\mathbf{s}_{i}$ and between $\mathbf{s}_{i}$ and $\mathbf{s}_{i+1}$, and $\mathcal{L}^{\star}$ is the original vortex configuration without the section between $\mathbf{s}_{i-1}$ and $\mathbf{s}_{i+1}$.

The number of discretization points, $N$, changes with time. As the simulation progresses, new discretization points are introduced to maintain the resolution along the vortex filament. If the separation between two points, $\mathbf{s}_{i}$ and $\mathbf{s}_{i+1}$, becomes greater than some threshold $\Delta \xi$, a new point is introduced at position $\mathbf{s}_{i^{\prime}}$ given by

$$
\mathbf{s}_{i^{\prime}}=\frac{1}{2}\left(\mathbf{s}_{i}+\mathbf{s}_{i+1}\right)+\left(\sqrt{R_{i^{\prime}}^{2}-\frac{1}{4} \ell_{i+1}^{2}}-R_{i^{\prime}}\right) \frac{\mathbf{s}_{i^{\prime}}^{\prime \prime}}{\left|\mathbf{s}_{i^{\prime}}^{\prime \prime}\right|},
$$

where $R_{i^{\prime}}=\left|\mathbf{s}_{i^{\prime}}^{\prime \prime}\right|^{-1}$. This approach preserves the curvature of the vortex filament, whilst ensuring that the separation of vortex points along a filament lies between $\Delta \xi / 2$ and $\Delta \xi$, where $\Delta \xi=0.05 \mathrm{~mm}$

All spatial derivatives are approximated using $4^{\text {th }}$ order finite difference schemes which account for varying mesh sizes along the vortex filaments. Time evolution is based on a $3^{\text {rd }}$ order Runge-Kutta scheme, with a time step equals to $\Delta t=5 \times 10^{-4} \mathrm{~s}$.

It is well known that if quantised vortices become close then they can reconnect [29]. If two discretization points become closer to each other than $\Delta \xi$, a numerical algorithm reconnects the two filaments subject to the criteria that the total length (as proxy for energy) decreases [30]. Self-reconnections (which can arise if a vortex filament has twisted back on itself) are treated in the same manner. Other reconnection algorithms exist [31], however in a previous study [32] we verified that in uniform counterflow important properties of the system were robust to the method of reconnection used.

All calculations are performed in a cuboid of size $D_{x} \times D_{y} \times D_{z}=4 \pi \mathrm{mm} \times 2 \mathrm{~mm} \times 4 \pi / 3 \mathrm{~mm}$. Periodic boundary conditions are used in the $x$ and $z$ directions, whereas solid boundaries are applied at $y= \pm D_{y} / 2$. In all simulations the counterflow lies parallel to the elongated $x$ direction. The boundaries are assumed to be perfectly smooth, hence no modelling of the pinning of vortices to any roughness on the boundaries is required. This is justified as the flow velocities considered here are much larger than the modest velocities required to de-pin quantised vortices from the boundary [22].

\section{NORMAL FLUID VELOCITY}

If we assume that the normal fluid is a laminar flow in the positive $x$ direction, trapped between solid boundaries at $y= \pm D_{y} / 2$ (assuming no-slip boundaries) then it is appropriate to take the following simple Poiseuille profile

$$
\mathbf{v}_{n}=\left(1-\frac{y^{2}}{h^{2}}\right) U_{c} \mathbf{e}_{\mathbf{x}}
$$

where $h=D_{y} / 2$ is the half-width of the channel. The relative motion of the two velocity fields, commonly denoted $v_{n s}$, is then simply given by $v_{n s}=2 U_{c} / 3$, the mean flow velocity along the channel. 

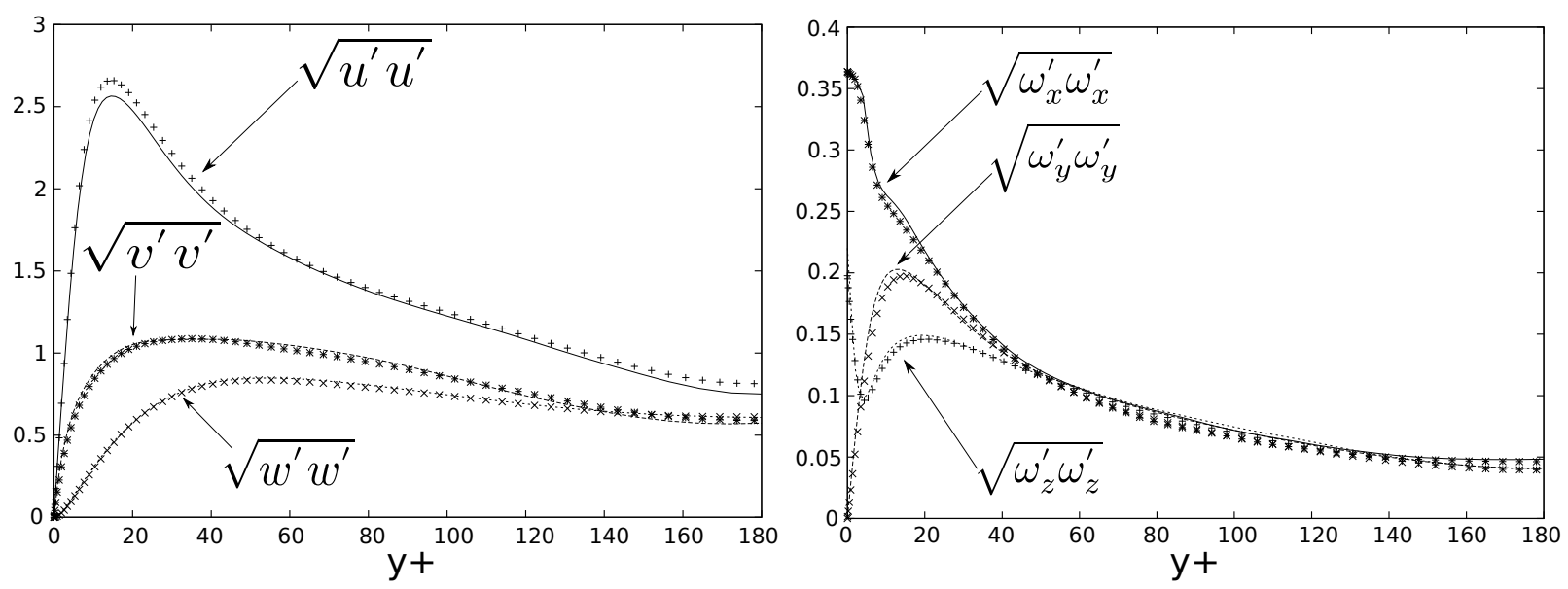

FIG. 1. Turbulent intensities (left) and r.m.s of the fluctuating vorticity components (right) in wall units. Lines: present simulation. Symbols: [36].

In the case of a turbulent channel flow the situation is clearly more complex. The governing equations, assuming an incompressible normal fluid $\left(\boldsymbol{\nabla} \cdot \mathbf{v}_{n}=0\right)$, are the Navier-Stokes equations

$$
\frac{\partial \mathbf{v}_{n}}{\partial t}=-\nabla p_{n}-\frac{1}{2}\left[\boldsymbol{\nabla}\left(\mathbf{v}_{n} \otimes \mathbf{v}_{n}\right)+\left(\mathbf{v}_{n} \cdot \nabla\right) \mathbf{v}_{n}\right]+\nu \nabla^{2} \mathbf{v}_{n}+\mathbf{f}
$$

where $p_{n}(\mathbf{x}, t)$ is the pressure field (for a fluid with a constant density $\rho_{n}=1$ ), $\nu$ is the kinematic viscosity, and $\mathbf{f}$ is an externally applied force.

Equations (6) are solved using the code Incompact3d [33]. This code is based on sixth-order compact finite difference schemes for the spatial differentiation and a third order Runge-Kutta scheme for the time integration. To treat the incompressibility condition, a fractional step method requires solving a Poisson equation. This equation is fully solved in spectral space, via the use of relevant 3D Fast Fourier Transforms. The pressure mesh is staggered from the velocity mesh by half a mesh to avoid spurious pressure oscillations. With the help of the concept of modified wave number [34], the divergence-free condition is ensured up to machine accuracy. Full details about the code can be found in Laizet \& Lamballais 35.

\section{A. Numerical parameters}

In order to investigate superfluid turbulence in a channel flow configuration, a Direct Numerical Simulation (DNS) of a turbulent channel flow is performed with a Reynolds number of $R e=U_{m} h / \nu=2793$ where $U_{m}$ is the bulk velocity and $h=D_{y} / 2$ is the half-width of the channel. Using the conventional notation where superscript + indicates a scaling based on the friction velocity $u_{\tau}$ and the kinematic velocity $\nu$, this Reynolds number leads to $h^{+} \approx 178.1$. The numerical domain is based on $n_{x} \times n_{y} \times n_{z}=128 \times 129 \times 84$ mesh nodes to discretize the computational domain $D_{x} \times D_{y} \times D_{z}=4 \pi \mathrm{mm} \times 2 \mathrm{~mm} \times 4 \pi / 3 \mathrm{~mm}$, with $x, y$ and $z$ being the longitudinal, normal to the wall and spanwise directions respectively. In the $y$-direction, a stretching is applied in order to concentrate grid points near the walls at $y= \pm h$ with the first adjacent point to the wall located at $y^{+}=1$ (see Laizet \& Lamballais [35] for further details about the stretching). Periodic boundary conditions are used in $x$ and $z$ directions while no-slip boundary conditions are imposed at the two walls $y= \pm h$. These boundary conditions are consistent with those applied to the quantised vortices.

In order to get a fully turbulent state, an initial random perturbation is superimposed on a Poiseuille profile

$$
\mathbf{v}_{n}=\left(1-\frac{y^{2}}{h^{2}}\right) U_{c} \mathbf{e}_{\mathbf{x}}
$$

In order to quickly obtain a fully turbulent state, a rotation in the $z$ direction is imposed in the early stage of the simulation with a Rossby number equal to $R o=U_{m} h / f=1 / 18$ where $f$ is the Coriolis frequency. 


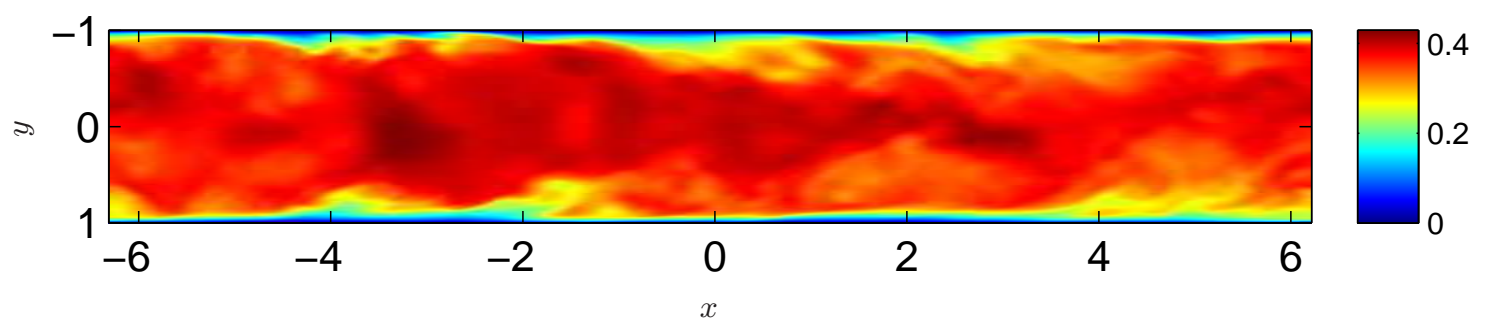

FIG. 2. Slice of the $x$ component of the turbulent normal fluid in the $x y$-plane at $z=0$.

The velocity field is then time stepped until a fully developed turbulent state is achieved; this is then validated against previous studies. Profiles of velocity and vorticity r.m.s obtained with our simulations are presented in figure [1. An excellent agreement with previous spectral DNS of Moser et al. [36] is found. Figure 2 shows the structure of the turbulent channel flow in the $x y$-plane at $z=0$.

Due to the high computational cost of computing the evolution of the quantised vortices and the turbulent normal fluid, we follow Kivotides [28] and use a frozen normal fluid, i.e. a single snapshot of the turbulent normal fluid is used meaning $\mathbf{v}_{n}$ is time independent in both the laminar and turbulent simulations. As pointed out in [28], this approach can be loosely justified at the low normal fluid Reynolds numbers use in this study, where the turnover time of eddies in the normal fluid is relatively slow. In addition in counterflow there is an anisotropy in the structure of the tangle [18, 24] leading to the vortices drifting with respect to the imposed superflow. Hence the vortices move through the computational domain, and so in the rest frame of the vortices the flow appears time dependent. In order to make comparisons between the laminar and turbulent cases we scale $\mathbf{v}_{n}$ such that $\left\langle\mathbf{v}_{n} \cdot \mathbf{e}_{\mathbf{x}}\right\rangle=v_{n s}=0.34 \mathrm{~cm} / \mathrm{s}$, where angled brackets denote spatial averaging.

\section{RESULTS}

We present the results of four numerical simulations, all initialised with 100 randomly oriented loops confined to the numerical domain, with a radius of $0.0597 \mathrm{~mm}$. These consist of simulations using a laminar (Poiseuille) normal fluid and the frozen DNS channel flow, at two different temperatures $\mathrm{T}=1.6 \mathrm{~K}\left(\alpha=0.097, \alpha^{\prime}=0.0161\right)$ and $\mathrm{T}=1.9 \mathrm{~K}$ $\left(\alpha=0.206, \alpha^{\prime}=0.00834\right)$. We monitor the evolution of the vortex line density $L=\Lambda / V$, where $V$ is the volume of the domain and $\Lambda=\int_{\mathcal{L}} d \xi$ is the total length of the vortex configuration. In all simulations after an initial transition, where a rapid growth in the vortex line density $L$ is observed, the system saturates to a non-equilibrium steady state, as it can be seen in Figure 3 Here energy extracted from the normal fluid (through the Donnelly-Glaberson instability) is balanced by energy dissipation due to vortex reconnections [15, 16].

At both temperatures we see that the simulations with a turbulent normal fluid saturate at a much higher vortex line density. Of obvious interest is how this difference can arise, and if any differences in the structure of the vortex tangle are apparent. It is also interesting to note that the vortex line density in the lower temperature $(\mathrm{T}=1.6 \mathrm{~K})$ laminar simulation is slightly higher than in the corresponding higher temperature simulation. Initially this may seem counterintuitive as the timescale for the growth of Kelvin waves by the Donnelly-Glaberson instability is inversely proportional to $\alpha \underline{37}$, and so it would seem reasonable to assume larger values of $\alpha$ would lead to larger values of $L$. However this discrepancy can also be explained by examining the structure of the tangle.

\section{A. Vortex line density versus counterflow velocity}

In his seminal works Vinen [15, 16] introduced a phenomenological model for the evolution of the vortex line density $L(t)$ in a homogeneous vortex tangle. In a steady state regime $(d L / d t=0)$ Vinen's equation yields the following scaling law

$$
\sqrt{L}=\gamma(T)\left(v_{n s}-v_{0}\right)
$$

where $v_{0}$ is an additional fitting parameter, and $\gamma$ is a temperature dependent parameter. The measurement of $\gamma$ and the verification of this relationship is well established in the literature, across both experimental [12, 38] and numerical [18, 39] studies. 


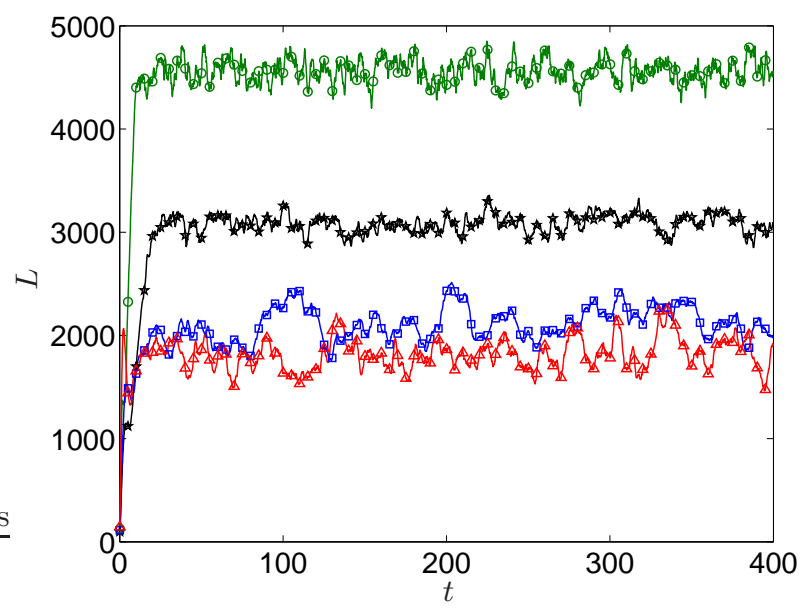

FIG. 3. The evolution of the vortex line density, $L\left(\mathrm{~cm}^{-2}\right)$, plotted in time, $t(\mathrm{~s})$. Solid (blue) line plotted with squares laminar $\mathbf{v}_{n}, \mathrm{~T}=1.6 \mathrm{~K}$; (red) line plotted with triangles - laminar $\mathbf{v}_{n}, \mathrm{~T}=1.9 \mathrm{~K}$; (black) line plotted with stars - turbulent $\mathbf{v}_{n}$, $\mathrm{T}=1.6 \mathrm{~K}$; (green) line plotted with circles - turbulent $\mathbf{v}_{n}, \mathrm{~T}=1.9 \mathrm{~K}$.

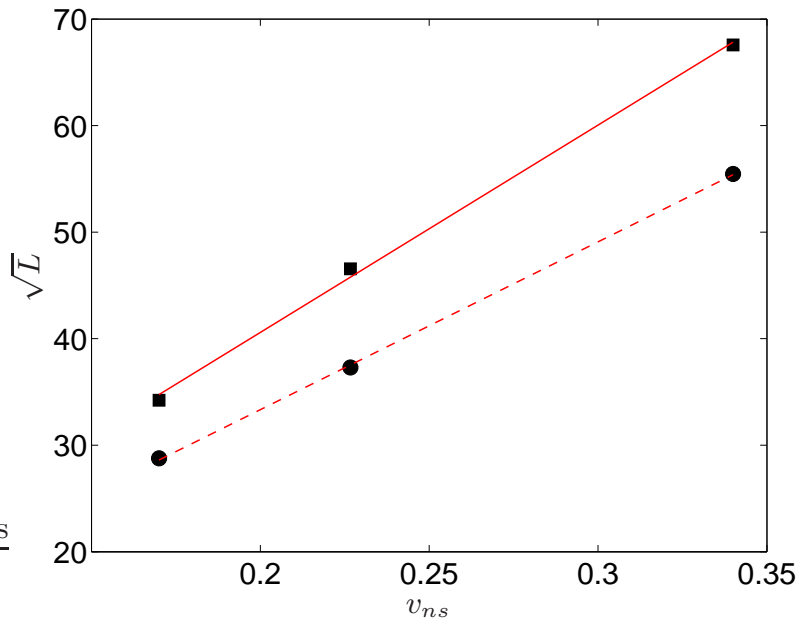

FIG. 4. The square root of the vortex line density $L\left(\mathrm{~cm}^{-2}\right)$ vs $v_{n s}(\mathrm{~cm} / \mathrm{s})$ for simulations with a turbulent normal fluid at $\mathrm{T}=1.6 \mathrm{~K}$ (circles) and $\mathrm{T}=1.9 \mathrm{~K}$ (squares). Lines of best fit are used to compute the scaling coefficient $\gamma\left(\mathrm{s} / \mathrm{cm}^{2}\right)$; we find $\gamma=195 \mathrm{~s} / \mathrm{cm}^{2}(1.9 \mathrm{~K})$ and $\gamma=157 \mathrm{~s} / \mathrm{cm}^{2}(1.6 \mathrm{~K})$.

However at a fixed temperature the value of $\gamma$ was found to dramatically increase when the applied heat flux (i.e. the counterflow velocity $v_{n s}$ ) became larger than some critical value. This led Tough [12] to categorised two turbulent regimes TI and TII. Using linear stability analysis Melotte \& Barenghi [21] suggested that transition to turbulence in the normal fluid was the underlying reason for the transition from the TI to TII regime. With the caveat of the steady imposed flow used in this study, we are in a position to shed some light on this problem, by computing $\gamma$ for the turbulent normal fluid, and comparing the results to experimental [12, 38] and numerical [18] results.

We perform further simulations using the turbulent (DNS) channel flow and rescaling the counterflow velocity to lower values of $v_{n s}=0.23 \mathrm{~cm} / \mathrm{s}$ and $0.17 \mathrm{~cm} / \mathrm{s}$, at both $\mathrm{T}=1.6 \mathrm{~K}$ and $1.9 \mathrm{~K}$. Figure 4 shows the familiar linear scaling of the square root of the vortex line density $L$ with the imposed counterflow velocity. The gradient of the line of best (least squares) fit to the data gives $\gamma=195 \mathrm{~s} / \mathrm{cm}^{2}(1.9 \mathrm{~K})$ and $\gamma=157 \mathrm{~s} / \mathrm{cm}^{2}(1.6 \mathrm{~K})$. These are significantly larger than the values quoted in Adachi et al. 18], where a laminar (constant) counterflow was imposed, and are comparable to experimental values [38], in the TII regime. Hence turbulence in the normal fluid is certainly capable of leading to larger values of $\gamma$, lending the first firm numerical evidence of a transition to turbulence in the TI to TII transition. 

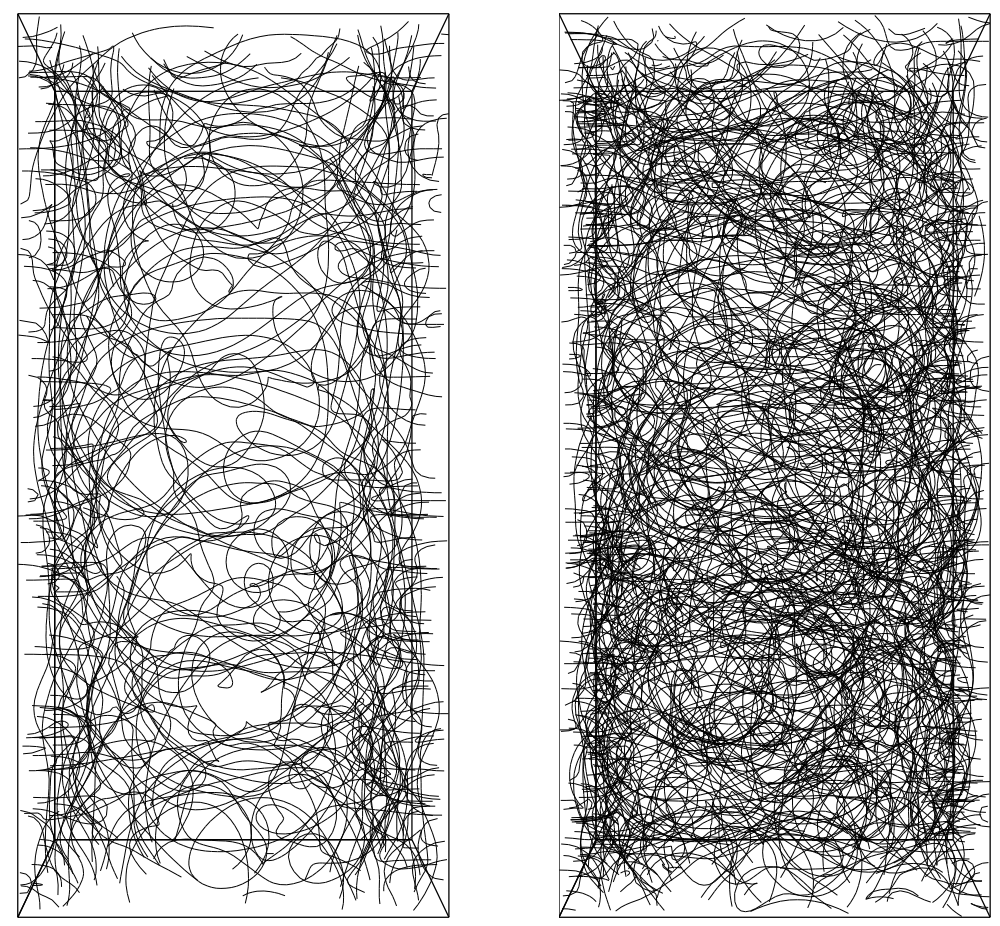

FIG. 5. Snapshots of the vortex configuration in the laminar (left) and turbulent (right) simulations, at $\mathrm{T}=1.9 \mathrm{~K}$, and $t=400 \mathrm{~s}$, plotted in the $y z$-plane. Notice the much higher vortex density in the turbulent case, as well as a more homogeneous distribution of vortices.

\section{B. Structure of the tangle}

Figure 5 displays snapshots of the vortex tangle when driven by a laminar (left) and turbulent normal fluid (right) in the higher temperature simulations $(\mathrm{T}=1.9 \mathrm{~K})$, plotted in the $y z$-plane. Clear differences in the spatial homogeneity of the tangles are visible. In order to quantify these differences we perform a course graining of the statistics of the tangle in time. A regular $16^{3}$ Cartesian mesh is defined in the computational volume. Within each discretization volume in this mesh, course grained information about the tangle is computed, including the total vortex line density within each sub-volume, the mean curvature and the mean velocity. We average this course grained mesh in time, over 3500 realisations, computed at 0.1 second intervals; subsequently we average over the two periodic directions $x$ and $z$ to find average measures as a function of $y$, i.e. across the channel. More formally we can define these quantities as,

$$
\begin{gathered}
L^{\prime}(y)=\left\langle\frac{1}{\mathcal{V}^{\prime}} \int_{\mathcal{L}^{\prime}} d \xi\right\rangle \\
\kappa^{\prime}(y)=\left\langle\int_{\mathcal{L}^{\prime}}\left|\mathbf{s}(\xi)^{\prime \prime}\right| d \xi / \int_{\mathcal{L}^{\prime}} d \xi\right\rangle \\
v_{x, y, z}^{\prime}(y)=\left\langle\int_{\mathcal{L}^{\prime}} \frac{d s_{x, y, z}}{d t} d \xi / \int_{\mathcal{L}^{\prime}} d \xi\right\rangle
\end{gathered}
$$

where $\mathcal{L}^{\prime}$ denotes the vortex configuration within the reduced volume, $\mathcal{V}^{\prime}$, each element of the Cartesian mesh encloses; angled brackets denote the temporal and spacial averaging procedure defined above. Clearly from Eq. (9) one can define an equivalent inter vortex spacing $\ell^{\prime}(y)=1 / \sqrt{L^{\prime}}$. Figure 6 (left) shows the course grained line density plotted along the channel. It is clear that with a laminar normal fluid the vortex line density is concentrated closer to the wall, in contrast to the turbulent simulations where the distribution of vortex density is more homogeneous, visible in the snapshots of the vortex tangles presented in Figure 5 

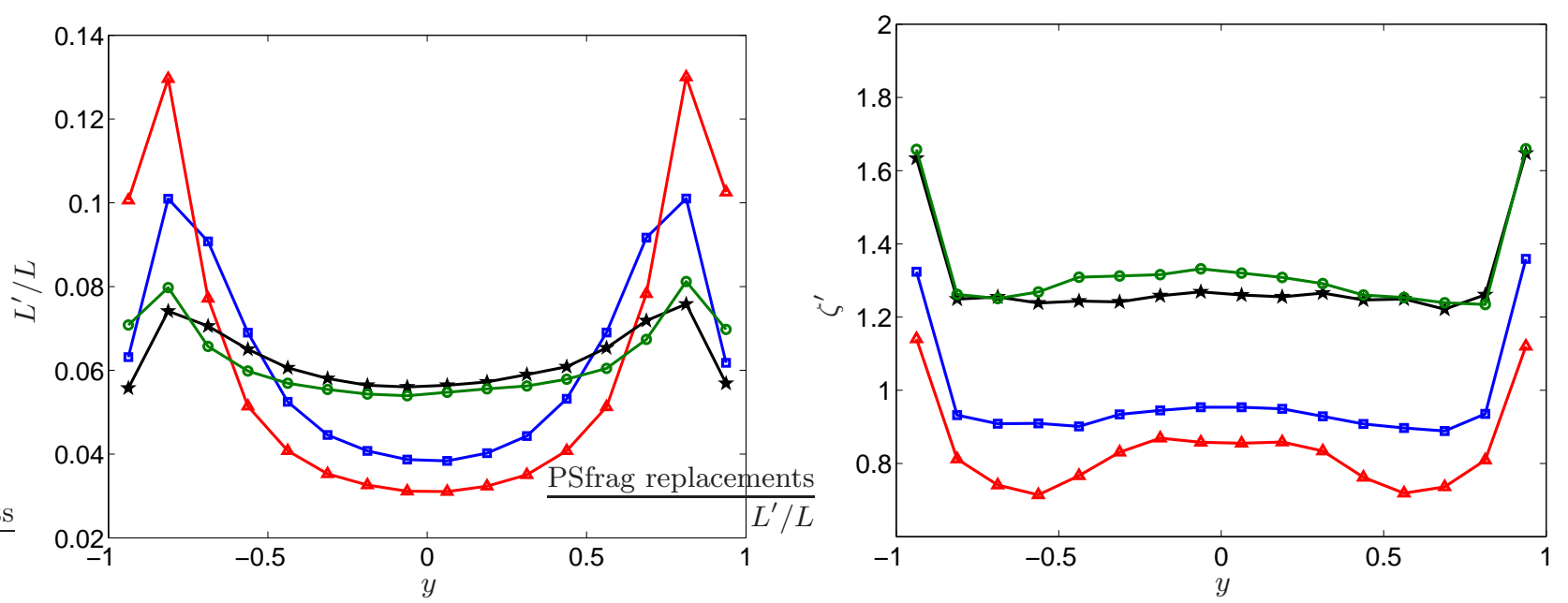

FIG. 6. Course grained statistics of the tangle plotted as function of the wall normal direction, $y$. Line style (colour) is as in Fig 3. The left panel displays the spatial distribution of the vortex line density $L^{\prime}(y)$, normalised by the total vortex line density $L$; the right panel displays $\zeta^{\prime}(y)$ the spatial distribution of the curvature $\kappa^{\prime}(y)$, scaled by the inter vortex spacing $\ell^{\prime}(y)$.

As we have discussed the steady state value of the vortex line density is a complex interplay between the energy transferred via the Donnelly-Glaberson (DG) instability and the energy dissipated due to vortex reconnections. The DG instability is the amplification of Kelvin waves (helical displacements of the vortex cores) by the component of the counterflow velocity parallel to the vortex, hence seed disturbances, wiggles along the vortices, are key for converting normal fluid energy to vortex length. This is balanced by the density of vortices which controls the rate of reconnection [40]. Therefore, a useful quantity to study is the dimensionless variable $\zeta=\kappa / \sqrt{L}=\kappa \ell$, with larger values providing an explanation for larger global vortex line densities. A course grained form, $\zeta^{\prime}$, of this quantity can readily be computed using Eqns. (9) \& (10). This quantity is displayed in Figure 6 (right). It can be seen that we find larger values corresponding to larger values of the global vortex line density $L$, plotted in Figure 3 . Therefore, one of the effects of turbulence in the normal fluid is to create higher curvature along the vortices, which promote the DG instability and allow a greater vortex line density to be reached. From inspection of Figure 6 (right) it is now perhaps clear why, in the laminar simulations, a marginally higher steady-state vortex line density is reached in the lower temperature simulation. As Kelvin waves are not damped as strongly at lower temperatures (due to reduced friction), vortex lines tend to have larger curvature, and so at least in these simulations a perhaps unexpected result is found.

Lending further weight to the importance of the curvature of the vortices is the fact that the profile of the average drift velocities of the vortices (see Equation 11) is very similar in all simulations. Figure 7 shows unscaled values of each of the components of $\mathbf{v}^{\prime}$ computed from the higher temperature $(\mathrm{T}=1.9 \mathrm{~K})$ turbulent normal fluid simulation, plotted as a function of $y$. Results from the other simulations are comparable with mean values of the $x$ and $y$ components of the vortices' velocity being comparable (in terms of magnitude) and the magnitude of the mean value of the $z$ component being orders of magnitude smaller. In order to compare between simulations we normalise each component of the course-grained velocity by the mean of the absolute value of the same quantity, plotting $v_{i}(y)^{\prime} / \overline{\left|v_{i}(y)^{\prime}\right|}$ (where $i=x, y, z$ and the overline denotes averaging in the $y$ direction) in Figure 8 . In all simulations we see the vortices in the centre of the channel move in the direction of the normal fluid, while being pushed to the walls of the channel by the $y$ component of the mutual friction. Slight differences in the mean $y$ component of the velocity between the turbulent and laminar simulations are visible, most likely due to different profiles of the mean streamwise velocity.

In all simulations it is interesting to note that although the mean $z$ component of the drift velocity is very small, it is not random as one might initially expect. This is probably due to the fact that small loops moving in the direction of the normal fluid increase in length (due to the DG instability) before reconnecting with the walls of the container leaving small hairpin vortices (see Figure 9) which are oppositely oriented on each side of the channel. If these vortices (initially moving in the $x$ direction) are preferentially twisted to lie in the $x y$-plane then on each side of the channel they would travel in opposite directions, as seems to be indicated by Figure 8

This near wall 'boundary layer' is also particularly interesting, if one examines the structure of the vortices here. Visible in the scaled course grained curvature plot (see Figure 6) is the noticeably higher curvature region close to the solid boundary. To probe the structure we plot all vortex lines which start and end on the same solid boundary. 


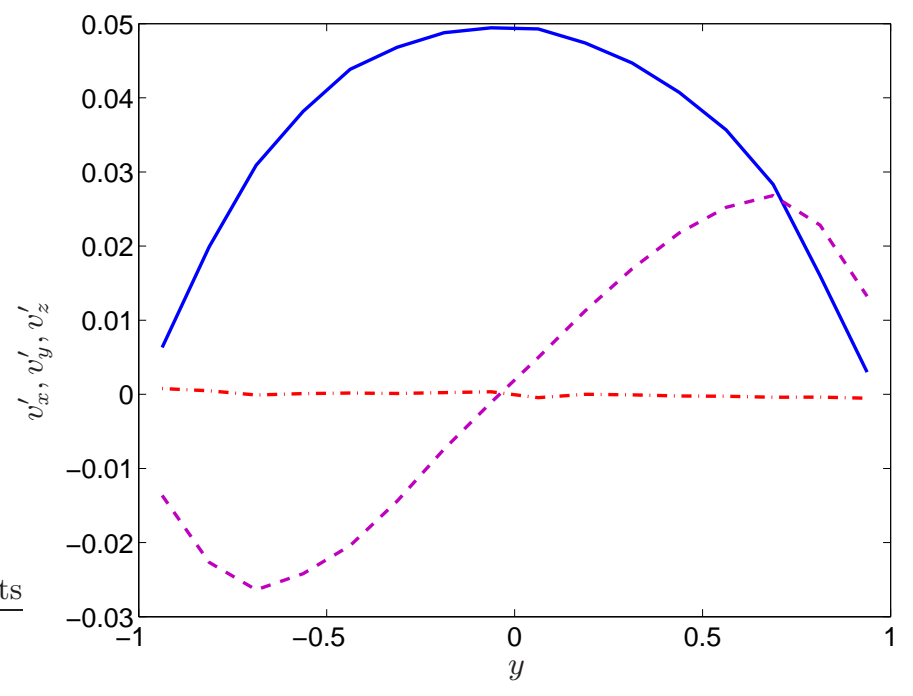

FIG. 7. Course grained components of the drift velocity of the vortices $\mathbf{v}_{i}^{\prime}(y)$, as defined in Eq. 11. The solid (blue) line represents $v_{x}^{\prime}$; (purple) dot-dashed $v_{y}^{\prime}$; (red) dot-dashed $v_{z}^{\prime}$. These results are computed from the higher temperature $(\mathrm{T}=1.9 \mathrm{~K})$ turbulent normal fluid simulation.
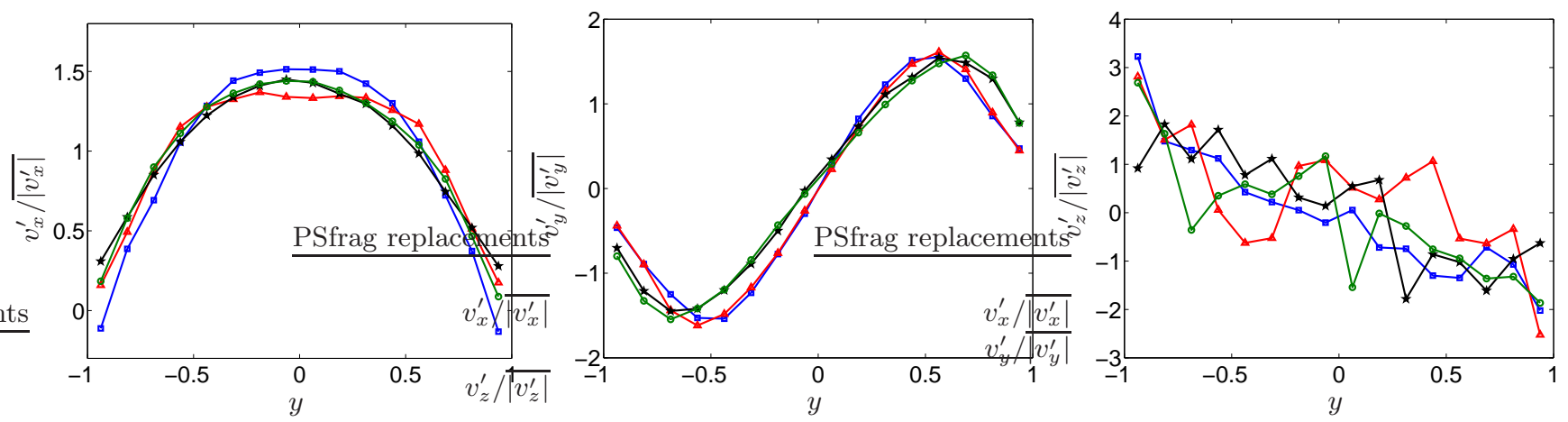

FIG. 8. Normalised course grained components of the drift velocity of the vortices $\mathbf{v}=d \mathbf{s} / d t$, as defined in Eq. 11 plotted for the four simulations (line style (colour) is as in Fig 3), the overbear denotes averaging in the $y$ direction.

These vortices (at three different times) are displayed in Figure 9, for the turbulent normal fluid simulations at $\mathrm{T}=1.9 \mathrm{~K}$. Results for the other simulations are consistent, with a varying number of loops depending on the density at the boundary. At least qualitatively their appearance is reminiscent of hairpin vortex structures often identified in turbulent boundary layers in classical fluids [41].

We also inspect the isotropy of the vortices. For each snapshot of the vortex tangles, in the saturated quasi-steady state, we compute the total vortex length parallel to each of the Cartesian directions, normalised by the total vortex line length and then averaged in time. More specifically we denote

$$
\Lambda_{i}=\left\langle\int_{\mathcal{L}} \mathbf{s}^{\prime} \cdot \mathbf{e}_{i} d \xi / \int_{\mathcal{L}} d \xi\right\rangle
$$

where $i=x, y, z$ and the angled brackets denote averaging in time. Figure 10 displays the variation of these statistics across the four simulations in the study; the results are constant with previous studies [18]. In all simulations the tangles are highly anisotropic with vortices lying in planes perpendicular to the counterflow. In addition the vortex configuration is more anisotropic at higher temperatures. Turbulence in the normal fluid leads to a marginal increase in isotropy of the tangle, which would probably be more pronounced with increasing Reynolds number. 


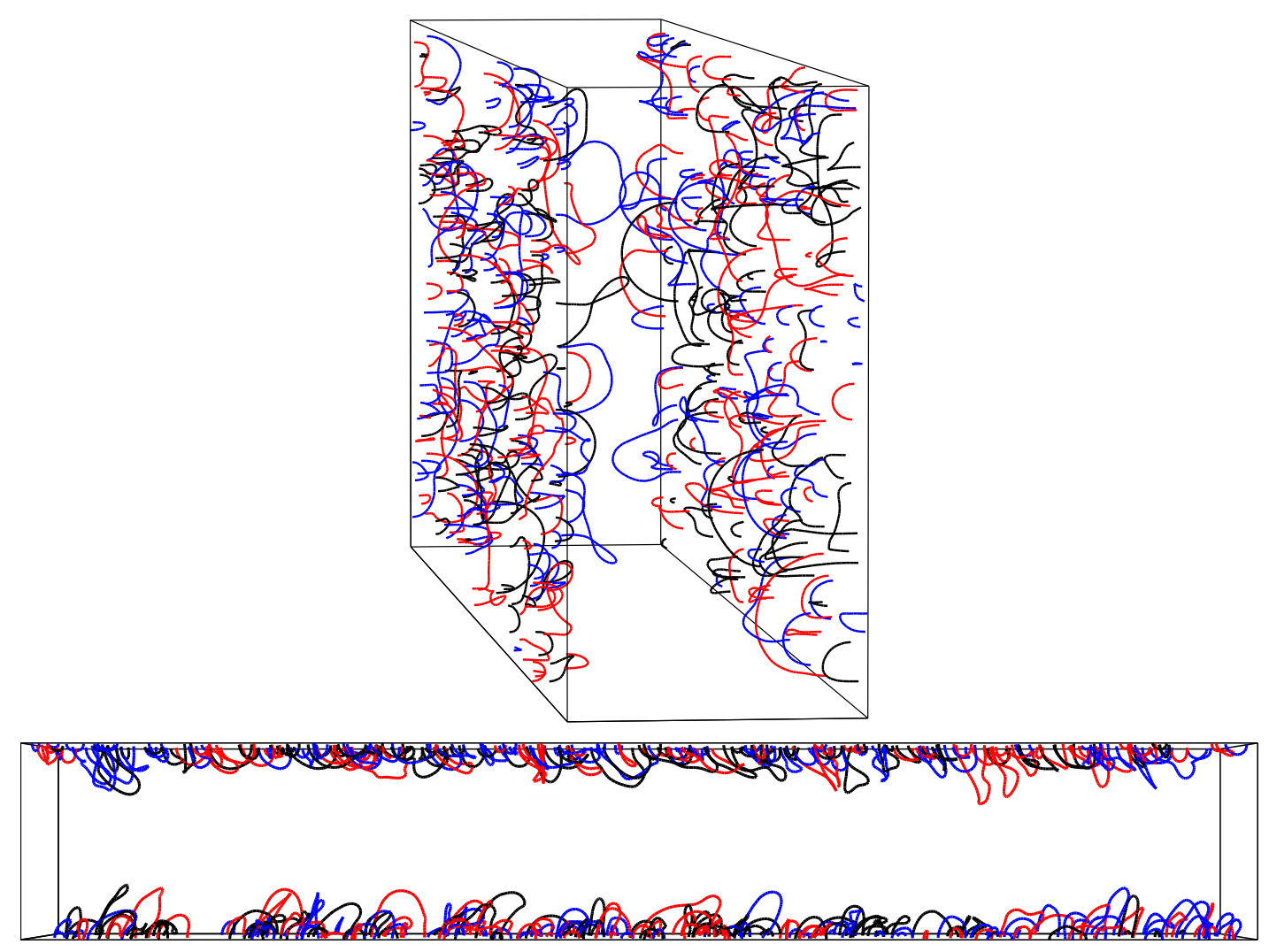

FIG. 9. The structure of vortices near the sold boundaries. Vortices which start and end on the same solid boundary are plotted for the simulation with a turbulent normal fluid at $\mathrm{T}=1.9 \mathrm{~K}$. Red lines are at $t=100 \mathrm{~s}$, black lines at $t=250 \mathrm{~s}$, and blue lines at $t=400 \mathrm{~s}$. The lower panel, plotted in the $x y$-plane shows that the vortex loops have a tendency to be angled 'downstream' (the normal fluid velocity flowing left to right here).

\section{Polarization of the tangle}

Following 42, 43] we further examine the structure of the vortex tangle through a measure of the vortex polarization. L'vov et al. [42], quantified the polarization of the tangle by defining the circulation, $\Gamma(R)$ over a contour of a twodimensional disc of radius $R$. Vortices intersect this disc, in both the positive and negative direction, and $\Gamma(R)$ is given by

$$
\Gamma(R)=\kappa\left(N_{+}-N_{-}\right)
$$

Assuming the following scaling

$$
\left\langle\Gamma^{2}\right\rangle=\kappa^{2}(R / \ell)^{\sigma}
$$

then L'vov et al. [42] showed that an unpolarized tangle has $\sigma=2$, and a tangle with the Kolmogorov $\left(k^{-5 / 3}\right)$ spectrum leads to $\sigma=8 / 3$. From the exponent $\sigma$, the polarisation of the tangle can then be defined as $P=\sigma / 2-1$. The value of $P$ depends on the spectral slope of the flow induced by the tangle, and so is an interesting and informative statistics to compute. We define a set of random discs in the $x z$-plane with radius $\ell \leq R \leq h$, at a fixed distances from the channel walls. This allows us to examine the polarisation as a function of the wall normal direction, following the analysis performed in $\$ \mathrm{IVB}$. We average our results over 10000 discs, at a given radius, for 3500 snapshots of the tangle in the steady-state regime; our results are displayed in Fig. 11. In all simulations we find that the tangle displays a small negative polarisation, i.e. there is a tendency for anti-parallel vortices to lie close to each other. It is worth commenting that L'vov et al. [42] state that the tangle in counterflow will be unpolarized $(P=0)$. One possible cause of negative polarisation are vortex reconnections, which dominate the dynamics of counterflow [25]. During a vortex reconnection the induced motion of the vortices tends to reduce any polarization, by turning the vortices as they approach. After reconnection, high curvature is created where vortices lie antiparallel to one another. 


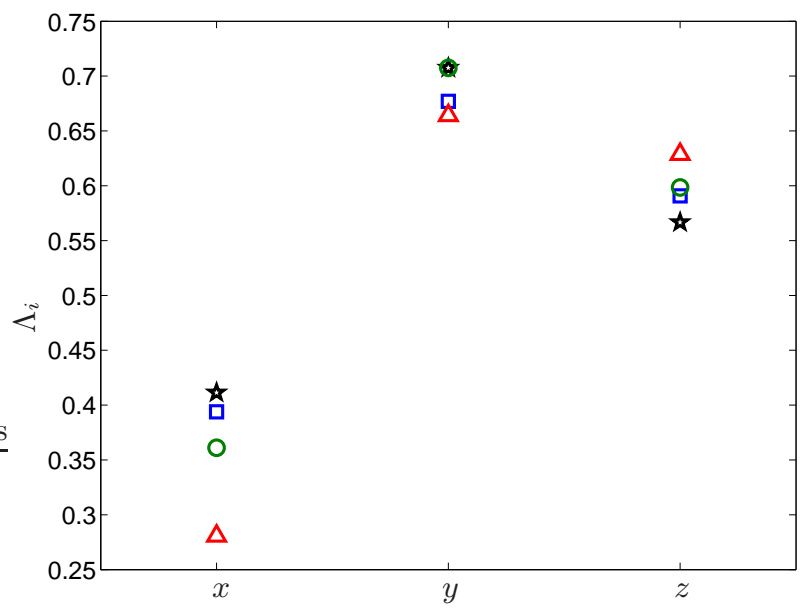

FIG. 10. Vortex length parallel to each of the Cartesian directions $\Lambda_{i}$, see Eq. (12), where $i=x, y, z$. (blue) Squares - laminar $\mathbf{v}_{n}, \mathrm{~T}=1.6 \mathrm{~K} ;\left(\right.$ red) triangles - laminar $\mathbf{v}_{n}, \mathrm{~T}=1.9 \mathrm{~K} ;$ (black) stars - turbulent $\mathbf{v}_{n}, \mathrm{~T}=1.6 \mathrm{~K} ;$ (green) circles - turbulent $\mathbf{v}_{n}$, $\mathrm{T}=1.9 \mathrm{~K}$.

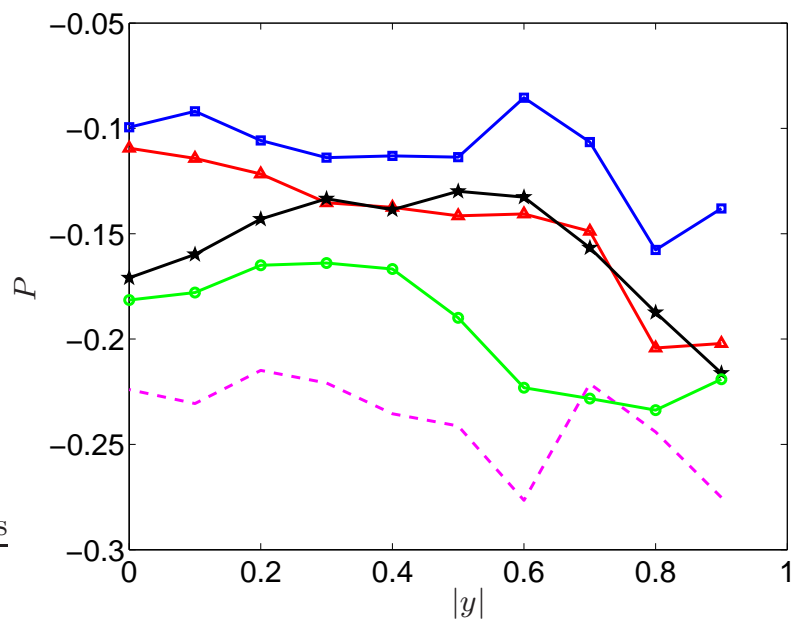

FIG. 11. The polarization of the vortex tangle computed from Eq. (14), using discs in the $x z$-plane, at a fixed distance from the channel walls $(|y|=1)$. The four curves marked with symbols are computed from the full vortex configurations (e.g. Fig. 55) of the four simulations discussed in the study (line style (colour) is as in Fig 3). The dashed (magenta) line is from the DNS simulation $(\mathrm{T}=1.9 \mathrm{~K})$, but only accounting for points which lie within $\ell / 2$ of another vortex segment.

Reconnections are more likely where the vortex line density is highest, which is closest to the walls, see Fig. 6. As we would expect, based on the above reasoning, in all simulations the largest magnitude of $P$ is also found closest to the walls.

We can probe this further, for the high temperature $(\mathrm{T}=1.9 \mathrm{~K})$ simulation performed with the turbulent normal fluid we repeat the above analysis, but only including points which lie within $\ell / 2$ of another vortex segment. Our reasoning is that these are likely places where a reconnection has taken place or is about to occur. We find this leads to the largest value of negative polarisation, with $P \approx-0.25$ across the channel. Hence the seemingly random tangles generated by counterflow are not completely random, and exhibit some organisation, one plausible cause is vortex reconnection. 

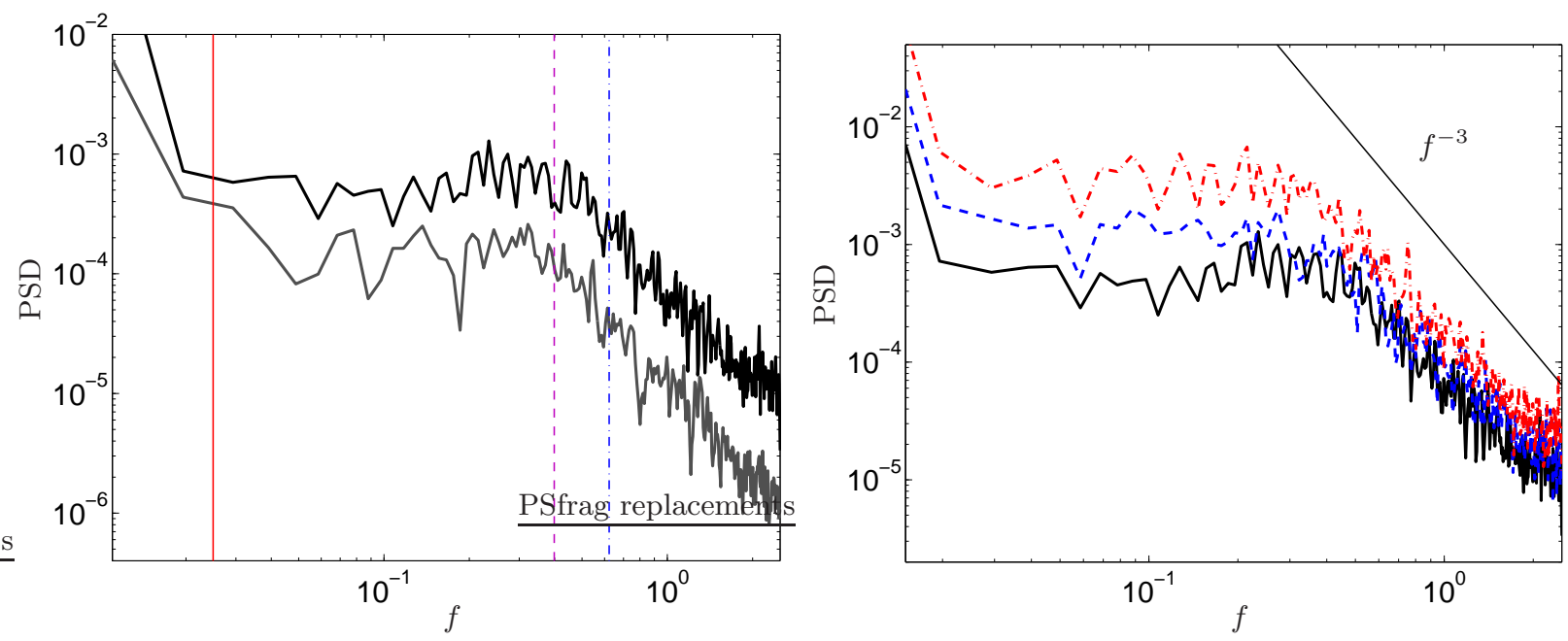

FIG. 12. Power spectral densities of fluctuations of the vortex line density within a volume of the computational domain (arbitrary units, scaled for display purposes) vs. $f\left(\mathrm{~s}^{-1}\right)$ computed during the saturated regime (50s $\left.\leq t \leq 400 \mathrm{~s}\right)$. (Left) For a comparison between the laminar (lower grey line) and turbulent (upper black line) simulations $(\mathrm{T}=1.9 \mathrm{~K})$ computed in a 1 mm cube at $x=y=z=0$. The solid vertical (red) line corresponds to the frequency $\Gamma / D_{y}^{2}$, the two dashed vertical lines on the right correspond to the frequencies $\Gamma / \ell^{2}$, dashed (purple) for the laminar simulation, dot-dashed (blue) for the turbulent simulation. (Right) PSDs of density fluctuations in the turbulent simulation $(\mathrm{T}=1.9 \mathrm{~K})$ computed from different volumes in the channel: black solid line - $1 \mathrm{~mm}$ cube at $x=y=z=0$; dashed (blue) line - $1 \mathrm{~mm}$ cube at $x=z=0, y=0.5 \mathrm{~mm}$; dot-dashed (red) line - $2 \mathrm{~mm}$ cube at $x=y=z=0$.

\section{Fluctuations of the vortex line density}

Studying the spectral properties of fluctuating quantities has proven to be a powerful technique in the analysis of turbulent flows. Hence, it is not surprising that a number of experimental studies (see Tough [12] for an overview) have analysed the power spectral density (PSD) of various quantities, most notably the fluctuations of $L$, in thermal counterflow. However as yet there is no consensus opinion on the form of the PSD, as a function of the frequency $f$. From heron we shall refer to $\operatorname{PSD}(f)$ as the power spectral density of the fluctuating component of the vortex line density, $\delta L$, where $L=L_{0}+\delta L$ is decomposed into a mean, $L_{0}$, and fluctuating part. An early study by Hoch et al. [44] observed a PSD which scaled as $f^{-1}$ at low frequencies, and $f^{-3}$ at high frequencies. This contrasts with Ostermeier et al. [45] and Barenghi et al. [46] who report a flat spectrum at low frequencies, but again with a spectrum consistent with $f^{-3}$ at higher frequencies (specifically [46] report $f^{-2.7 \pm 0.4}$ ). It is worth noting that if one considers the phenomenological model of Vinen [15, 16], then a PSD, flat in the low frequency region, and decaying as $f^{-2}$ is expected [46].

Our goal here is not to perform a detailed test of theory, or debate the validity of experimental results, but to compare the PSD of $\delta L$ (visible in Figure 3) between the turbulent and laminar simulations. To this end we use a 'virtual' probe a $1 \mathrm{~mm}^{3}$ cube centred at the origin. After the initial transient we compute the total vortex line density, within this region, to generate a time series of $\delta L$. Using Welch's method a PSD is computed from this signal. In Figure 12 (left) we compare the results of the laminar and turbulent simulations at $\mathrm{T}=1.9 \mathrm{~K}$ (results at $\mathrm{T}=1.6 \mathrm{~K}$ are consistent). A flat region, within the 'inertial range' of the turbulence is visible, with a power law decay at higher frequencies. In Figure 12 (right) we show that changing the location of the 'probe' to the boundaries, or increasing the size of the volume has no effect on the features of the PSD. We also show that the scaling of the decay at high frequency is consistent with $f^{-3}$.

The fact that the Reynolds number of the normal fluid is relatively modest, as well as the fact that we take a frozen snapshot, could be responsible for the fact that we observed no difference in the nature of the PSDs between the turbulent and laminar simulations. In contrast at high Reynolds numbers in mechanically pumped coflow [47] a PSD scaling as $f^{-5 / 3}$ was observed. This result can be explained as randomly oriented vortex lines are advected like passive scalars which explains a $f^{-5 / 3}$ scaling in Kolmogorov turbulence [48] . Further work is required to determine whether a Kolmogorov scaling is observed in counterflow turbulence when the Reynolds number of the normal fluid is large. 


\section{CONCLUSIONS}

To summarise we have presented clear evidence that turbulence in the normal fluid can sustain a substantially higher vortex line density for a given mean flow rate in thermal counterflow. We computed the scaling coefficient $\gamma$, and found a marked increase in its value when the normal fluid is turbulent. This offers the first numerical evidence that the transition from TI to TII turbulent states is due to turbulence in the normal fluid [12, 21]. Turbulence in the normal fluid leads to higher curvature along the vortices, particularly in the centre of the channel, this allows more energy to be extracted from the normal fluid through the Donnelly-Glaberson instability, and supports the observed increase in vortex line density. Through quantification of the polarization of counterflow generated vortex configurations we find that the tangle has a degree of negative polarization; the amount of polarisation appears to be correlated with the vortex line density. We offer evidence that vortex reconnections are a possible cause of negative polarization. We also examined the power spectral densities of the fluctuations of the vortex line density; our findings are consistent with experimental and theoretical results. At low Reynolds numbers considered in this study we observe no discernible difference between the PSDs computed from the laminar and turbulent simulations. In future studies we plan to investigate the effect of increasing flow rate with a time-dependent turbulent counterflow.

\section{ACKNOWLEDGMENTS}

AWB acknowledges the support of the Carnegie Trust, and fruitful discussions with Carlo Barenghi and Sergey Nazarenko. We are grateful for the comments of the anonymous referees who's comments added to the scope and depth of the paper.

[1] L. Skrbek and K. R. Sreenivasan, "Developed quantum turbulence and its decay," Phys. Fluids 24, 011301 (2012).

[2] W. F. Vinen and J. J. Niemela, "Quantum turbulence," J. Low Temp. Phys. 128, 167 (2002).

[3] J. Maurer and P. Tabeling, "Local investigation of superfluid turbulence," EPL (Europhysics Letters) 43, 29 (1998).

[4] J. Salort, C. Baudet, B. Castaing, B. Chabaud, F. Daviaud, T. Didelot, P. Diribarne, B. Dubrulle, Y. Gagne, F. Gauthier, et al., "Turbulent velocity spectra in superfluid flows," Phys. Fluids 22, 125102 (2010).

[5] C. Nore, M. Abid, and M. E. Brachet, "Kolmogorov turbulence in low-temperature superflows," Phys. Rev. Lett. 78, 3896 (1997).

[6] T. Araki, M. Tsubota, and S. K. Nemirovskii, "Energy spectrum of superfluid turbulence with no normal-fluid component," Phys. Rev. Lett. 89, 145301 (2002).

[7] A. W. Baggaley, C. F. Barenghi, A. Shukurov, and Y. A. Sergeev, "Coherent vortex structures in quantum turbulence," EPL (Europhysics Letters) 98, 26002 (2012).

[8] J. Salort, B. Chabaud, E. Lvque, and P.-E. Roche, "Energy cascade and the four-fifths law in superfluid turbulence," EPL (Europhysics Letters) 97, 34006 (2012).

[9] L. Skrbek, A. V. Gordeev, and F. Soukup, "Decay of counterflow He II turbulence in a finite channel: Possibility of missing links between classical and quantum turbulence," Phys. Rev. E 67, 047302 (2003).

[10] P. Walmsley and A. Golov, "Quantum and quasiclassical types of superfluid turbulence," Phys. Rev. Lett. 100, 245301 (2008).

[11] R. Donnelly, Quantized Vortices in Helium II, no. v. 2 in Cambridge Studies in American Literature and Culture (Cambridge University Press, 1991), ISBN 9780521324007.

[12] J. Tough, in Progress of Low Temperature Physics Vol. VIII, edited by D. Brewer (North-Holland Publications, Amsterdam, 1982).

[13] W. F. Vinen, "Mutual friction in a heat current in liquid helium II. I. Experiments on steady heat currents," Proceedings of the Royal Society of London. Series A. Mathematical and Physical Sciences 240, 114 (1957).

[14] W. F. Vinen, "Mutual friction in a heat current in liquid helium II. II. Experiments on transient effects," Proceedings of the Royal Society of London. Series A. Mathematical and Physical Sciences 240, 128 (1957).

[15] W. F. Vinen, "Mutual friction in a heat current in liquid helium II. III. Theory of the mutual friction," Proceedings of the Royal Society of London. Series A. Mathematical and Physical Sciences 242, 493 (1957).

[16] W. F. Vinen, "Mutual friction in a heat current in liquid helium II. IV. Critical heat currents in wide channels," Proceedings of the Royal Society of London. Series A. Mathematical and Physical Sciences 243, 400 (1958).

[17] C. F. Barenghi, A. V. Gordeev, and L. Skrbek, "Depolarization of decaying counterflow turbulence in He II," Phys. Rev. E 74, 026309 (2006).

[18] H. Adachi, S. Fujiyama, and M. Tsubota, "Steady-state counterflow quantum turbulence: Simulation of vortex filaments using the full Biot-Savart law," Phys. Rev. B 81, 104511 (2010).

[19] L. Galantucci, C. Barenghi, M. Sciacca, M. Quadrio, and P. Luchini, "Turbulent superfluid profiles in a counterflow channel," J. Low Temp. Phys. 162, 354 (2011). 
[20] W. Guo, S. B. Cahn, J. A. Nikkel, W. F. Vinen, and D. N. McKinsey, "Visualization study of counterflow in superfluid ${ }^{4}$ He using metastable helium molecules," Phys. Rev. Lett. 105, 045301 (2010).

[21] D. Melotte and C. Barenghi, "Normal fluid velocity profile and transition from t-1 to t-2 state of superfluid turbulence," J. Low Temp. Phys. 113, 573 (1998).

[22] K. W. Schwarz, "Three-dimensional vortex dynamics in superfluid ${ }^{4}$ He: Line-line and line-boundary interactions," Phys. Rev. B 31, 5782 (1985).

[23] R. J. Donnelly and C. F. Barenghi, "The observed properties of liquid helium at the saturated vapor pressure," J. Phys. Chem. Ref. Data 27, 1217 (1998).

[24] K. W. Schwarz, "Three-dimensional vortex dynamics in superfluid he 4: Homogeneous superfluid turbulence," Phys. Rev. B 38, 2398 (1988).

[25] A. W. Baggaley, L. K. Sherwin, C. F. Barenghi, and Y. A. Sergeev, "Thermally and mechanically driven quantum turbulence in helium II," Phys. Rev. B 86, 104501 (2012).

[26] K. Morris, J. Koplik, and D. W. I. Rouson, "Vortex locking in direct numerical simulations of quantum turbulence," Phys. Rev. Lett. 101, 015301 (2008).

[27] D. Kivotides, J. C. Vassilicos, C. F. Barenghi, M. A. I. Khan, and D. C. Samuels, "Quantum signature of superfluid turbulence," Phys. Rev. Lett. 87 (2001).

[28] D. Kivotides, "Coherent structure formation in turbulent thermal superfluids," Phys. Rev. Lett. 96, 175301 (2006).

[29] M. S. Paoletti, M. E. Fisher, K. R. Sreenivasan, and D. P. Lathrop, "Velocity statistics distinguish quantum turbulence from classical turbulence," Phys. Rev. Lett. 101, 154501 (2008).

[30] M. Leadbeater, T. Winiecki, D. C. Samuels, C. F. Barenghi, and C. S. Adams, "Sound emission due to superfluid vortex reconnections," Phys. Rev. Lett. 86, 1410 (2001).

[31] L. Kondaurova and S. K. Nemirovskii, "Full Biot-Savart numerical simulation of vortices in He II," J. Low Temp. Phys. 138, $555(2005)$.

[32] A. Baggaley, "The sensitivity of the vortex filament method to different reconnection models," J. Low Temp. Phys. 168, 18 (2012).

[33] This open source code is now available at http://code.google.com/p/incompact3d/.

[34] S. K. Lele, "Compact finite difference schemes with spectral-like resolution," J. Comp. Phys. 103, 16 (1992).

[35] S. Laizet and E. Lamballais, "High-order compact schemes for incompressible flows: a simple and efficient method with the quasi-spectral accuracy," J. Comp. Phys. 228(16), 5989 (2009).

[36] R. D. Moser, J. Kim, and N. N. Mansour, "Direct numerical simulation of turbulent channel flow up to Re $\tau=590$," Phys. Fluids 11, 943 (1999).

[37] M. Tsubota, C. F. Barenghi, T. Araki, and A. Mitani, "Instability of vortex array and transitions to turbulence in rotating helium II," Phys. Rev. B 69, 134515 (2004).

[38] S. Babuin, M. Stammeier, E. Varga, M. Rotter, and L. Skrbek, "Quantum turbulence of bellows-driven ${ }^{4}$ he superflow: Steady state," Phys. Rev. B 86, 134515 (2012).

[39] A. Baggaley and C. Barenghi, "Tree method for quantum vortex dynamics," Journal of Low Temperature Physics 166, 3 (2012).

[40] C. F. Barenghi and D. C. Samuels, "Scaling laws of vortex reconnections," J. Low Temp. Phys. 136, 281 (2004).

[41] S. K. Robinson, "Coherent motions in the turbulent boundary layer," Annual Review of Fluid Mechanics 23, 601 (1991).

[42] V. S. L'vov, S. V. Nazarenko, and O. Rudenko, "Bottleneck crossover between classical and quantum superfluid turbulence," Phys. Rev. B 76, 024520 (2007).

[43] A. W. Baggaley, "The importance of vortex bundles in quantum turbulence at absolute zero," Physics of Fluids (1994present) 24, 055109 (2012).

[44] H. Hoch, L. Busse, and F. Moss, "Noise from vortex-line turbulence in He II," Phys. Rev. Lett. 34, 384 (1975).

[45] R. Ostermeier, M. Cromar, P. Kittel, and R. Donnelly, "Fluctuations in turbulent He II counterflow," Phys. Lett. A 77, 321 (1980).

[46] C. F. Barenghi, C. E. Swanson, and R. J. Donnelly, "Induced vorticity fluctuations in counterflowing He II," Phys. Rev. Lett. 48, 1187 (1982).

[47] P.-E. Roche, P. Diribarne, T. Didelot, O. Franais, L. Rousseau, and H. Willaime, "Vortex density spectrum of quantum turbulence," EPL (Europhysics Letters) 77, 66002 (2007).

[48] A. W. Baggaley, J. Laurie, and C. F. Barenghi, "Vortex-density fluctuations, energy spectra, and vortical regions in superfluid turbulence," Phys. Rev. Lett. 109, 205304 (2012). 